\title{
Interview with Maria Chudnovsky
}

Toufik Mansour

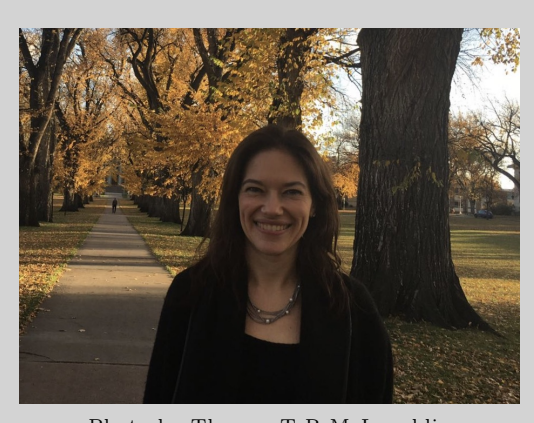

Photo by Theresa T-R McLaughlin

Maria Chudnovsky received a B.Sc. in mathematics from the Technion, and her Ph.D. in 2003 from Princeton University under the supervision of Paul Seymour. After postdoctoral research at the Clay Mathematics Institute, she became an assistant professor at Princeton University in 2005, and moved to Columbia University in 2006. By 2014, she was the Liu Family Professor of Industrial Engineering and Operations Research at Columbia. In 2015, she returned to Princeton as a professor of mathematics. In 2004, Chudnovsky was named one of the "Brilliant 10" by Popular Science magazine. Her work on the strong perfect graph theorem won for her and her co-authors the 2009 Fulkerson Prize. In 2013 she was awarded the MacArthur grant. Professor Chudnovsky is a leading expert on graph theory and contributions to the field including the proof of the strong perfect graph theorem (with Neil Robertson, Paul Seymour, and Robin Thomas), the first polynomial-time algorithm for recognizing perfect graphs, and of a structural characterization of the claw-free graphs.

Mansour: Professor Chudnovsky, first of all we would like to thank you for accepting this interview. Would you tell us broadly what combinatorics is?

Chudnovsky: I think there are almost as many answers to this question as there are combinatorialists... I usually say that combinatorics is the study of discrete patterns.

Mansour: What do you think about the development of the relations between combinatorics and the rest of mathematics?

Chudnovsky: I think this is a wide two-way street. Obviously many questions in all areas of math lead to understanding a pattern of objects; and conversely combinatorial questions can be encoded as instances of other problems, to which solutions are known by methods from different fields. Examples of that are topological methods, algebraic methods, the probabilistic method, and many others.

Mansour: What have been some of the main goals of your research?

Chudnovsky: I study structural graph the- ory. What that means is that you give me a class of graphs with certain properties, and I try to come up with a description of its members. If I succeed, then I can answer questions about your class of graphs, because I now completely understand how they are built. However, often coming up with explicit structure is too hard (or maybe impossible), so then there are compromises. First you ask me a question about a class of graphs, then I try to understand enough about how they are built-in order to answer your question. I mostly work on classes of graphs that are defined by forbidding certain induced subgraphs.

Mansour: We would like to ask you about your formative years. What were your early experiences with mathematics? Did that happen under the influence of your family, or some other people?

Chudnovsky: I grew up in a home where mathematics was treated with utmost respect; it was the most important subject in school, and in the world... I also went to a special

The authors: Released under the CC BY-ND license (International 4.0), Published: November 3, 2020

Toufik Mansour is a professor of mathematics at the University of Haifa, Israel. His email address is tmansour@univ.haifa.ac.il 
math school in St Petersburg (school \#30; I am guessing more than a few of your readers are its graduates), which was a fantastic place to get an introduction to the subject. After my family came to Israel (I was 13 at the time) I studied math in the Columbia program at the Leo Baeck High School in Haifa; I also attended the Technion Math Circle. All of these were amazing experiences, each taught me different kinds of math. More importantly, each taught me how to see the beauty of mathematics and gave me the first taste of the experience of thinking about a problem.

Chudnovsky: Were there specific problems that made you first interested in combinatorics?

Chudnovsky: Absolutely! If six people come to a party, then either there are three who know each other or three who do not. Five are not enough. I heard this problem when I was about 14 or 15; and I have never been the same person again.

Mansour: What was the reason you chose Princeton University for your Ph.D. and your advisor, Paul Seymour?

Chudnovsky: I asked my M.Sc. adviser Ron Aharoni where to apply for my Ph.D. He recommended a few places. I applied there, and got into some and not others. The rest is history...

Mansour: How was the mathematics at Princeton at that time?

Chudnovsky: It was (and I hope still is) an unbelievably vibrant and active place. There was a charge in the air (or at least that is how it felt to me as a first-year graduate student) of all these amazing conjectures about to be conquered. I was thrilled to be allowed to be a part of it.

Mansour: Your Ph.D. work was on perfect graphs and the result, known as the strong perfect graph theorem, is considered by many experts in the field as one of the most important recent developments in mathematics. Would you tell us about perfect graphs and your result?

Chudnovsky: A graph $G$ is perfect if for every induced subgraph $H$ of $G$, the chromatic number of $H$ equals the clique number of $H$. (The clique number is an obvious lower bound for the chromatic number; if this lower bound achieved for all induced subgraphs of $G$, then $G$ is perfect). Perfect graphs were defined by the French mathematician Claude Berge in 1961, and they have some amazing properties and connections. First of all, many seemingly unrelated theorems in graph theory and combinatorics can be rephrased as stating that graphs in a certain family are perfect. Secondly, there are algorithmic problems that are hard (NPcomplete) in general, but that can be solved efficiently (in polynomial time) if the input graph is perfect. It also turns out that being perfect is invariant under taking complements (the complement $G^{c}$ of $G$ is the graph with the same vertex set as $G$, and two vertices are adjacent in $G$ if and only if they are non-adjacent in $G^{c}$ ). Berge conjectured this invariance when he defined perfect graphs, calling it "The Weak Perfect Graph Conjecture"; László Lovász proved it in $1972^{1}$. In addition to this conjecture, Berge also proposed a list of minimal (under taking induced subgraphs) graphs that are not perfect. This list consisted of all odd cycles of length at least five, and their complements; and the "Strong Perfect Graph Conjecture" of Berge states that a graph if perfect if and only if it does not contain (as an induced subgraph) any of the graphs in this list. One direction of this conjecture is an easy exercise: it is not hard to see that for each graph in the list, the chromatic number is strictly bigger than the clique number, and therefore they cannot appear as induced subgraph in a perfect graph. The other direction turned out to be a lot harder; it remained open for over 40 years, attracting a lot of attention, and leading to the development of a lot of beautiful directions in graph theory and combinatorial optimization. In 2003 Paul Seymour, Neil Robertson, Robin Thomas and I proved this conjecture and that is the Strong Perfect Graph Theorem.

Mansour: Would you comment about why the strong perfect graph theorem has been so influential?

Chudnovsky: I think it is because so much theory was built motivated by finding its solution. Also, as I said earlier, many facts in several fields can be stated in the language of perfection, so having an equivalent, and easier to verify notion is very helpful under many circumstances.

\footnotetext{
${ }^{1}$ See https://www.sciencedirect.com/science/article/pii/0095895672900457?via\%3Dihub
} 
Mansour: What would guide you in your research? A general theoretical question or a specific problem?

Chudnovsky: There is no one answer, and most of the time it is somewhere in between.

Mansour: When you are working on a problem, do you feel that something is true even before you have the proof?

Chudnovsky: Definitely. Sometimes it then turns out to be false ...

Mansour: What are the top three open questions in your list?

- The Erdös-Hajnal Conjecture: This is a conjecture that states that for every graph $H$ there is an $\epsilon(H) \in(0,1)$ such that every $n$-vertex graph with no induced subgraph isomorphic to $H$ has a clique or a stable set of size at least $n^{\epsilon(H)}$.

- Hadwigger's Conjecture: This one says that a graph that does not contains the complete graph on $t+1$ vertices as a minor is $t$-colorable.

- A combinatorial algorithm to find the largest clique in a perfect graph that runs in time polynomial in the number of vertices of the input. This problem of finding a largest clique in a perfect graph is known to be polynomial-time solvable (this is a result of Grotschel, Lovász and Schrijver), but the algorithm uses techniques from combinatorial optimization, such as the ellipsoid method. I would like to find an algorithm that can be formulated entirely in terms of the graph.

Mansour: What kind of mathematics would you like to see in the next ten-to-twenty years as the continuation of your work?

Chudnovsky: I would like to see more graph structure theorems, and algorithms based on them. I also hope that we will be able to understand better the behavior of the chromatic number of graphs with certain induced subgraphs forbidden.

Mansour: What would you say about some of the major directions in graph theory for the next two decades?

Chudnovsky: This is too hard to answer; I will pass on this one.

Mansour: Do you think that there are core or mainstream areas in mathematics? Are some topics more important than others?

Chudnovsky: I definitely think that some topics are more important than other, but only time will tell which ones they are.
Mansour: Are you also interested in some enumerative combinatorial questions? Do enumerative techniques play a role in your research?

Chudnovsky: There are many beautiful questions there, but I have not worked on them.

Mansour: What do you think about the distinction between pure and applied mathematics that some people focus on? Is it meaningful at all in your own case? How do you see the relationship between so-called "pure" and "applied" mathematics?

Chudnovsky: I do not really believe in pure and applied mathematics; only in mathematics and its applications.

Mansour: What advice would you give to young people thinking about pursuing a research career in mathematics?

Chudnovsky: There is zero reasons not to try. If you succeed, you will have a wonderful professional life. If not, you can get a great job outside of the pure research world using the skills you have accumulated, and again be very happy with your career. Doing mathematics teaches one to think clearly and critically, and that is a sought after commodity in every area of intellectual pursuit.

Mansour: While we see that there are more women in science and technology fields today than ever before, bias still affects women in their scientific careers. What do you think about this issue?

Chudnovsky: I think things have been steadily getting better, and I am very hopeful. It is a pleasure to see more and more women at conferences and workshops; I hope that within my lifetime a day will come when we will be able to take it for granted. The other day my seven-year-old son asked me: Mommy, why did people once think that girls are not good in math? I hope this is a reflection of what we will see 20 years from now.

Mansour: Would you tell us about your interests besides mathematics?

Chudnovsky: I love art and reading. I love walking around beautiful cities.

Mansour: Before we close this interview with one of the foremost experts in combinatorics, we would like to ask some more specific mathematical questions. You have worked on several graph theoretic problems throughout your career. What kind of graph questions are your 


\section{favorite?}

Chudnovsky: I like questions where there is an underlying phenomenon that you can understand, and then use this understanding to derive answers to questions. But I suspect that this is true for most people doing research.

Mansour: How would you describe doing research in graph theory? Does someone need to have a strong intuition or formidable technical abilities to solve difficult questions in the field? Chudnovsky: I will answer this in short: yes; you need both.

Mansour: Settling algorithmic complexity of some graph-theoretic questions is also very important and difficult. Would you tell us about your related works and point out some future directions?

Chudnovsky: I see many algorithmic results as a good concise way to "package" a lot of information about a certain class of graphs. I know this, and this, and that.. and you can not really write it as a theorem. But because of this, this and that I can efficiently approximate the clique number - and suddenly you have an interesting result to tell people about. Most of my algorithmic result have that flavor: I can prove enough about the structure of the input that certain algorithms follow.

Mansour: One of the main tools you used to prove the strong perfect graph theorem was a technical result, as you called the "wonderful lemma" (first proved by F. Roussel and P. Rubio $^{2}$ ). In 2018, you presented a very elegant yet shorter proof of this lemma. Why do you call it "wonderful lemma"?

Chudnovsky: Because it is a wonderful powerful tool that allows you to generalize some very simple facts about perfect graphs to much deeper statements. It is also used all the time in the proof of The Strong Perfect Graph Theorem.

Mansour: Would you tell us about your thought process for the proof of one of your favorite results? How did you become interested in that problem? How long did it take you to figure out a proof? Did you have a "eureka moment"?

Chudnovsky: When I try to prove that $A$ implies $B$, I usually try to come up with the description of the world where $A$ holds, and where $B$ follows. Then I try to prove that my picture is correct. Sometimes it is not, so I try to modify the world I have been imagining. And so I go around in what feels like "concentric circles" until either I find a solution, or I give up. Sometimes I cannot prove everything I imagined about $A$, but enough to get $B$.

Mansour: Graph coloring problems enjoy many practical applications as well as theoretical challenges and it is still a very active field of research. Would you briefly describe the main points in such problems and comment on some future directions?

Chudnovsky: One aspect of graph coloring is something called " $\chi$-boundedness". A class $\mathcal{C}$ of graphs, closed under taking induced subgraphs, is called $\chi$-bounded if there is a function $f_{\mathcal{C}}$ such that for every $G \in \mathcal{C}$ we have $\chi(G) \leq f_{\mathcal{C}}(\omega(G))$ (here $\chi(G)$ denotes the chromatic number of $G$, and $\omega(G)$ is the size of largest clique in $G$ ). The function $f_{\mathcal{C}}$ is called a $\chi$-bounding function for $\mathcal{C}$. Thus perfect graphs are a $\chi$-bounded class with the identity being a $\chi$-bounding function. The class of all graph is not $\chi$-bounded: there are several constructions of graphs with no clique of size three, and with arbitrarily large chromatic numbers. So it is a deep and elegant question to ask: which graph classes are $\chi$-bounded? There has been a lot of progress on this recently, and I hope there is more to come.

There are also beautiful algorithmic questions. In general, it is NP-complete to compute (or even approximate) the chromatic number of a graph, and even to answer if a graph is colorable with a fixed number $k$ of colors. But what if some graph $H$ is forbidden as an induced subgraph? It turns out that usually that does not help: as long as some component of $H$ is not a path, the problem remains NP-complete. So what about taking $H$ to be a path on $t$ vertices? Now for every $k$ and $t$ there is a problem: what is the complexity deciding if a graph with no induced path on $t$ vertices can be $k$-colored? And we know the answer if $k \geq 4$ or $t \leq 7$. The only open case is: what is the complexity of 3-coloring graphs with no induced $t$-vertex path when $t \geq 8$ ? Now there are variations: instead of excluding one graph $H$, exclude several. Instead of asking for an efficient coloring algorithm, ask for a list of minimal obstructions. There are a lot

${ }^{2}$ See https://www.sciencedirect.com/science/article/pii/S0095895601920441 
of lovely questions there.

Mansour: Do you think that one day someone or a group of researchers will be able to present a non-computer-assisted proof for the four-color theorem?

Chudnovsky: I really would not bet one way or another. On the one hand, I think there are more and more methods available that may help. On the other hand, I think our acceptance of computer-assisted proofs is growing, and so I am not sure how much motivation there will be to continue looking for a computer-free proof.

Mansour: What is the Erdös-Hajnal conjecture? Is there an exciting recent development related to this conjecture?

Chudnovsky: This conjecture states that for every graph $H$ there is an $\epsilon(H) \in(0,1)$ such that every $n$-vertex graph with no induced subgraph isomorphic to $H$ has a clique or a stable set of size at least $n^{\epsilon(H)}$. There have been a lot of developments on variants of this conjecture. What if instead of excluding one graph $H$, we excluded $H$ and its complement $H^{c}$ ? Or exclude a family of graphs? What if we try to play with the function in the conclusion? Ask for something slightly smaller that $n^{\epsilon}(H)$, but still big enough that it will be asymptotically different from the regime when nothing is excluded (then the correct function is $\log (n))$. Or we can formulate a stronger conclusion, and ask what needs to be excluded to achieve it. These are all beautiful and interesting questions; trying to think about them deepens our understanding of graphs with forbidden induced subgraphs. It is quite likely that what we learn from this is much more important than the exact asymptotic behavior of the function...

Mansour: Is there a specific problem you have been working on for many years? What progress have you made?

Chudnovsky: The Erdős-Hajnal conjecture, and the problem of computing the maximum clique size in a perfect graph.

Mansour: Professor Maria Chudnovsky, I would like to thank you for this very interesting interview on behalf of the journal Enumerative Combinatorics and Applications. 